Article

\title{
On a New Half-Discrete Hilbert-Type Inequality Involving the Variable Upper Limit Integral and Partial Sums
}

\author{
Jianquan Liao ${ }^{1}$, Shanhe $\mathrm{Wu}^{2, *}$ and Bicheng Yang ${ }^{1}$ \\ 1 Department of Mathematics, Guangdong University of Education, Guangzhou 510303, Guangdong, China; \\ liaojianquan@gdei.edu.cn (J.L.); bcyang@gdei.edu.cn (B.Y.) \\ 2 Department of Mathematics, Longyan University, Longyan 364012, Fujian, China \\ * Correspondence: shanhewu@163.com
}

Received: 5 January 2020; Accepted: 7 February 2020; Published: 10 February 2020

Abstract: In this paper we establish a new half-discrete Hilbert-type inequality involving the variable upper limit integral and partial sums. As applications, an inequality obtained from the special case of the half-discrete Hilbert-type inequality is further investigated; moreover, the equivalent conditions of the best possible constant factor related to several parameters are proved.

Keywords: weight coefficient; Euler-Maclaurin summation formula; half-discrete Hilbert-type inequality; partial sum; variable upper limit integral

\section{Introduction}

If $p>1, \frac{1}{p}+\frac{1}{q}=1, a_{m}, b_{n} \geq 0,0<\sum_{m=1}^{\infty} a_{m}^{p}<\infty$ and $0<\sum_{n=1}^{\infty} b_{n}^{q}<\infty$, then the famous Hardy-Hilbert's inequality with the best possible constant factor $\frac{\pi}{\sin (\pi / p)}$ reads as follows ([1], Theorem 315):

$$
\sum_{m=1}^{\infty} \sum_{n=1}^{\infty} \frac{a_{m} b_{n}}{m+n}<\frac{\pi}{\sin (\pi / p)}\left(\sum_{m=1}^{\infty} a_{m}^{p}\right)^{\frac{1}{p}}\left(\sum_{n=1}^{\infty} b_{n}^{q}\right)^{\frac{1}{q}}
$$

In [2], an extension of (1) was established by introducing parameters $\lambda_{i} \in(0,2](i=1,2)$, $\lambda_{1}+\lambda_{2}=\lambda \in(0,4]$, that is

$$
\sum_{m=1}^{\infty} \sum_{n=1}^{\infty} \frac{a_{m} b_{n}}{(m+n)^{\lambda}}<B\left(\lambda_{1}, \lambda_{2}\right)\left[\sum_{m=1}^{\infty} m^{p\left(1-\lambda_{1}\right)-1} a_{m}^{p}\right]^{\frac{1}{p}}\left[\sum_{n=1}^{\infty} n^{q\left(1-\lambda_{2}\right)-1} b_{n}^{q}\right]^{\frac{1}{q}}
$$

where the constant factor $B\left(\lambda_{1}, \lambda_{2}\right)$ is the best possible, and

$$
B(u, v)=\int_{0}^{\infty} \frac{t^{u-1}}{(1+t)^{u+v}} d t(u, v>0)
$$

is the beta function.

In [3], by applying inequality (2) and the Abel's summation by parts formula, Adiyasuren et al. gave a new inequality with the kernel $\frac{1}{(m+n)^{\lambda}}$ involving partial sums. The Hardy-Hilbert's inequality (1) and its integral analogues play an important role in analysis and its applications ([4-15]). 
In 1934, a half-discrete Hilbert-type inequality was given as follows ([1], Theorem 351): Assuming that $K(t)(t>0)$ is a decreasing function, and

$$
p>1, \frac{1}{p}+\frac{1}{q}=1,0<\phi(s)=\int_{0}^{\infty} K(t) t^{s-1} d t<\infty,
$$

$a_{n} \geq 0$ such that $0<\sum_{n=1}^{\infty} a_{n}^{p}<\infty$, then we have the following inequality:

$$
\int_{0}^{\infty} x^{p-2}\left(\sum_{n=1}^{\infty} K(n x) a_{n}\right)^{p} d x<\phi^{p}\left(\frac{1}{q}\right) \sum_{n=1}^{\infty} a_{n}^{p}
$$

Rassias, Yang, and Krnić et al. presented some extensions of inequality (4) in [16-20].

Hong and Wen [21] showed the equivalent statements of the extensions of (1) with the best possible constant factor related to several parameters. Some similar results relating to the extensions of inequalities (2) and (4) were given in [22-27].

Recently, Yang and Wu et al. [28,29] gave a reverse half-discrete Hardy-Hilbert's inequality and an extended Hardy-Hilbert's inequality. For these inequalities, the equivalent statements of the best possible constant factor related to several parameters were also discussed therein.

Following the way of $[2,4,21]$, the aim of this paper is to establish a new half-discrete Hilbert-type inequality involving the variable upper limit integral and partial sums via the kernel $\frac{1}{\left(x+n^{\alpha}\right)^{\lambda}}$. With regard to the obtained inequality, the equivalent conditions of the best possible constant factor related to several parameters are also proved.

\section{Some Lemmas}

In what follows, we suppose that $p>1, \frac{1}{p}+\frac{1}{q}=1, \lambda \in(0,4], \alpha \in(0,1], \lambda_{1} \in(0, \lambda+1), \lambda_{2} \in$ $\left(0, \frac{2}{\alpha}-1\right] \cap(0, \lambda+1)$,

$$
k_{\lambda}\left(\lambda_{i}\right):=B\left(\lambda_{i}, \lambda-\lambda_{i}\right)(i=1,2),
$$

where the beta function $B(u, v)(u, v>0)$ is defined by (3). For a nonnegative real function $f$ and a sequence of nonnegative real numbers $\left\{a_{n}\right\}_{n=1}^{\infty}$, we define the following variable upper limit integral

$$
F(x):=\int_{0}^{x} f(t) d t(x \geq 0)
$$

and the partial sums

$$
A_{n}:=\sum_{k=1}^{n} a_{k}(n \in \mathrm{N}=\{1,2, \cdots\}),
$$

satisfying $F(x)=o\left(e^{t x}\right), A_{n}=o\left(e^{t n^{\alpha}}\right)(t>0 ; x, n \rightarrow \infty)$ such that

$$
0<\int_{0}^{\infty} x^{-p\left(\frac{\lambda-\lambda_{2}}{p}+\frac{\lambda_{1}}{q}\right)-1} F^{p}(x)<\infty \text { and } 0<\sum_{n=1}^{\infty} n^{q\left[1-\alpha\left(1+\frac{\lambda-\lambda_{1}}{q}+\frac{\lambda_{2}}{p}\right)\right]-1} A_{n}^{q}<\infty
$$

Lemma 1. (i) ([5], (2.2.3)) If $g(t)$ is a positive real function and $g \in C^{3}(0, \infty)$ such that $(-1)^{i} \frac{d^{i}}{d t^{i}} g(t)>0, t \in$ $[m, \infty)(m \in N)$ with $g^{(i)}(\infty)=0(i=0,1,2,3)$ and $P_{i}(t), B_{i}(i \in N)$ are the Bernoulli functions and the Bernoulli numbers of $i$-order, then we have

$$
\int_{m}^{\infty} P_{2 q-1}(t) g(t) d t=-\varepsilon_{q} \frac{B_{2 q}}{2 q} g(m)\left(0<\varepsilon_{q}<1 ; q=1,2, \cdots\right) .
$$


In particular, for $q=1$, in view of $B_{2}=\frac{1}{6}$, we have

$$
-\frac{1}{12} g(m)<\int_{m}^{\infty} P_{1}(t) g(t) d t<0
$$

for $q=2$, in view of $B_{4}=-\frac{1}{30}$, we have

$$
0<\int_{m}^{\infty} P_{3}(t) g(t) d t<\frac{1}{120} g(m) .
$$

(ii) ([5], (2.2.16)) If $h(t)$ is a positive real function, $h \in C^{3}[m, \infty), h^{(i)}(\infty)=0(i=0,1,2,3)$, then the Euler-Maclaurin summation formulas hold:

$$
\begin{aligned}
& \sum_{k=m}^{\infty} h(k)=\int_{m}^{\infty} h(t) d t+\frac{1}{2} h(m)+\int_{m}^{\infty} P_{1}(t) h^{\prime}(t) d t \\
& \int_{m}^{\infty} P_{1}(t) h^{\prime}(t) d t=-\frac{1}{12} h^{\prime}(m)+\frac{1}{6} \int_{m}^{\infty} P_{3}(t) h^{\prime \prime \prime}(t) d t
\end{aligned}
$$

where $P_{i}(t)(i=1,3)$ are the Bernoulli functions.

Lemma 2. For $\alpha \in(0,1], s \in(0,6], s_{2} \in\left(0, \frac{2}{\alpha}\right] \cap(0, s), k_{s}\left(s_{2}\right)=B\left(s_{2}, s-s_{2}\right)$, let us define the following weight coefficient:

$$
\omega_{\alpha}\left(s_{2}, x\right):=\alpha \cdot x^{s-s_{2}} \sum_{n=1}^{\infty} \frac{1}{\left(x+n^{\alpha}\right)^{s}} n^{\alpha s_{2}-1}\left(x \in \mathrm{R}_{+}=(0, \infty)\right)
$$

Then the following inequalities hold:

$$
0<k_{s}\left(s_{2}\right)\left(1-O\left(\frac{1}{x^{s_{2}}}\right)\right)<\omega_{\alpha}\left(s_{2}, x\right)<k_{s}\left(s_{2}\right),
$$

where $O\left(\frac{1}{x^{2}}\right):=\frac{1}{k_{s}\left(s_{2}\right)} \int_{0}^{\frac{1}{x}} \frac{u^{s_{2}-1}}{(1+u)^{s}} d u>0$.

Proof. For fixed $x>0$, we define the real function $g_{x}(t)$ as follows:

$$
g_{x}(t):=\frac{\alpha t^{\alpha s_{2}-1}}{\left(x+t^{\alpha}\right)^{s}}(t>0)
$$

By using (9), we have

$$
\begin{aligned}
\sum_{n=1}^{\infty} g_{x}(n) & =\int_{1}^{\infty} g_{x}(t) d t+\frac{1}{2} g_{x}(1)+\int_{1}^{\infty} P_{1}(t) g^{\prime}{ }_{x}(t) d t \\
& =\int_{0}^{\infty} g_{x}(t) d t-h(x),
\end{aligned}
$$

where

$$
h(x):=\int_{0}^{1} g_{x}(t) d t-\frac{1}{2} g_{x}(1)-\int_{1}^{\infty} P_{1}(t) g_{x}^{\prime}(t) d t
$$


We obtain $-\frac{1}{2} g_{x}(1)=\frac{-\alpha}{2(x+1)^{s}}$. Integrating by parts, it follows that

$$
\begin{aligned}
& \int_{0}^{1} g_{x}(t) d t=\alpha \int_{0}^{1} \frac{t^{\alpha s_{2}-1}}{\left(x+t^{\alpha}\right)^{s}} d t \stackrel{u=t^{\alpha}}{=} \int_{0}^{1} \frac{u^{s_{2}-1}}{(x+u)^{s}} d u \\
= & \frac{1}{s_{2}} \int_{0}^{1} \frac{d u^{s_{2}}}{(x+u)^{s}}=\left.\frac{1}{s_{2}} \frac{u^{s_{2}}}{(x+u)^{s}}\right|_{0} ^{1}+\frac{s}{s_{2}} \int_{0}^{1} \frac{u^{s_{2}}}{(x+u)^{s+1}} d u \\
= & \frac{1}{s_{2}} \frac{1}{(x+1)^{s}}+\frac{s}{s_{2}\left(s_{2}+1\right)} \int_{0}^{1} \frac{d u^{s_{2}+1}}{(x+u)^{s+1}} \\
> & \frac{1}{s_{2}} \frac{1}{(x+1)^{s}}+\frac{s}{s_{2}\left(s_{2}+1\right)}\left[\frac{u^{s_{2}+1}}{(x+u)^{s+1}}\right]_{0}^{1}+\frac{s(s+1)}{s_{2}\left(s_{2}+1\right)(x+1)^{s+2}} \int_{0}^{1} u^{s_{2}+1} d u \\
= & \frac{1}{s_{2}} \frac{1}{(x+1)^{s}}+\frac{\lambda}{s_{2}\left(s_{2}+1\right)} \frac{1}{(x+1)^{s+1}}+\frac{s(s+1)}{s_{2}\left(s_{2}+1\right)\left(s_{2}+2\right)} \frac{1}{(x+1)^{s+2}},
\end{aligned}
$$

and

$$
\begin{aligned}
& -g^{\prime}{ }_{x}(t)=-\frac{\alpha\left(\alpha s_{2}-1\right) t^{\alpha s_{2}-2}}{\left(x+t^{\alpha}\right)^{s}}+\frac{\alpha^{2} s t^{\alpha+\alpha s_{2}-2}}{\left(x+t^{\alpha}\right)^{s+1}} \\
= & -\frac{\alpha\left(\alpha s_{2}-1\right) t^{\alpha s_{2}-2}}{\left(x+t^{\alpha}\right)^{s}}+\frac{\alpha^{2} s\left(x+t^{\alpha}-x\right) t^{\alpha s_{2}-2}}{\left(x+t^{\alpha}\right)^{s+1}} \\
= & \frac{\alpha\left(\alpha s-\alpha s_{2}+1\right) t^{\alpha s_{2}-2}}{\left(x+t^{\alpha}\right)^{s}}-\frac{\alpha^{2} s x t^{\alpha s_{2}-2}}{\left(x+t^{\alpha}\right)^{s+1}} .
\end{aligned}
$$

For $0<s_{2} \leq \frac{2}{\alpha}, 0<\alpha \leq 1, s_{2}<s \leq 6$, we have

$$
(-1)^{i} \frac{\partial^{i}}{\partial t^{i}}\left[\frac{t^{\alpha s_{2}-2}}{\left(x+t^{\alpha}\right)^{s}}\right]>0,(-1)^{i} \frac{\partial^{i}}{\partial t^{i}}\left[\frac{t^{\alpha s_{2}-2}}{\left(x+t^{\alpha}\right)^{s+1}}\right]>0(i=0,1,2,3) .
$$

By (7)-(10), we obtain

$$
\begin{aligned}
& \alpha\left(\alpha s-\alpha s_{2}+1\right) \int_{1}^{\infty} P_{1}(t) \frac{t^{\alpha s_{2}-2}}{\left(x+t^{\alpha}\right)^{s}} d t>-\frac{\alpha\left(\alpha s-\alpha s_{2}+1\right)}{12(x+1)^{s}}, \\
& -\alpha^{2} x s \int_{1}^{\infty} P_{1}(t) \frac{t^{\alpha s_{2}-2}}{\left(x+t^{\alpha}\right)^{s+1}} d t \\
= & \frac{\alpha^{2} x s}{12(x+1)^{s+1}}-\frac{\alpha^{2} x s}{6} \int_{1}^{\infty} P_{3}(t)\left[\frac{t^{\alpha s_{2}-2}}{\left(x+t^{\alpha}\right)^{s+1}}\right]_{t}^{\prime \prime} d t \\
> & \frac{\alpha^{2} x s}{12(x+1)^{s+1}}-\frac{\alpha^{2} x s}{720}\left[\frac{t^{\alpha s_{2}-2}}{\left(x+t^{\alpha}\right)^{s+1}}\right]_{t=1}^{\prime \prime} \\
> & \frac{\alpha^{2}(x+1-1) s}{12(x+1)^{s+1}}-\frac{\alpha^{2}(x+1) s}{720}\left[\frac{(s+1)(s+2) \alpha^{2}}{(x+1)^{s+3}}+\frac{\alpha(s+1)\left(5-\alpha-2 \alpha s_{2}\right)}{(x+1)^{s+2}}+\frac{\left(2-\alpha s_{2}\right)\left(3-\alpha s_{2}\right)}{(x+1)^{s+1}}\right] \\
= & \frac{\alpha^{2} s}{12(x+1)^{s}}-\frac{\alpha^{2} s}{12(x+1)^{s+1}} \\
& \quad-\frac{\alpha^{2} s}{720}\left[\frac{(s+1)(s+2) \alpha^{2}}{(x+1)^{s+2}}+\frac{\alpha(s+1)\left(5-\alpha-2 \alpha s_{2}\right)}{(x+1)^{s+1}}+\frac{\left(2-\alpha s_{2}\right)\left(3-\alpha s_{2}\right)}{(x+1)^{s}}\right] .
\end{aligned}
$$

Then we have

$$
\begin{aligned}
h(x)>\frac{1}{(x+1)^{s}} h_{1}+\frac{\lambda}{(x+1)^{s+1}} h_{2}+\frac{s(s+1)}{(x+1)^{s+2}} h_{3}, \\
h_{1}:=\frac{1}{s_{2}}-\frac{\alpha}{2}-\frac{\alpha-\alpha^{2} s_{2}}{12}-\frac{\alpha^{2} s\left(2-\alpha s_{2}\right)\left(3-\alpha s_{2}\right)}{720}, \\
h_{2}:=\frac{1}{s_{2}\left(s_{2}+1\right)}-\frac{\alpha^{2}}{12}-\frac{\alpha^{3}(s+1)\left(5-\alpha-2 \alpha s_{2}\right)}{720}
\end{aligned}
$$

and

$$
h_{3}:=\frac{1}{s_{2}\left(s_{2}+1\right)\left(s_{2}+2\right)}-\frac{\alpha^{4}(s+2)}{720} .
$$

We obtain

$$
h_{1} \geq \frac{1}{s_{2}}-\frac{\alpha}{2}-\frac{\alpha-\alpha^{2} s_{2}}{12}-\frac{s \alpha^{2}\left(2-\alpha s_{2}\right)\left(3-\alpha s_{2}\right)}{720}=\frac{g\left(s_{2}\right)}{720 s_{2}}
$$

where for $\sigma \in\left(0, \frac{2}{\alpha}\right]$,

$$
g(\sigma):=720-\left(420 \alpha+6 s \alpha^{2}\right) \sigma+\left(60 \alpha^{2}+5 s \alpha^{3}\right) \sigma^{2}-s \alpha^{4} \sigma^{3} .
$$


Namely, $g$ was previously defined as a function. We obtain that for $\alpha \in(0,1], s \in(0,6]$,

$$
\begin{aligned}
g^{\prime}(\sigma) & =-\left(420 \alpha+6 s \alpha^{2}\right)+2\left(60 \alpha^{2}+5 s \alpha^{3}\right) \sigma-3 \alpha^{4} \sigma^{2} \\
& \leq-420 \alpha-6 s \alpha^{2}+2\left(60 \alpha^{2}+5 s \alpha^{3}\right) \frac{2}{\alpha} \\
& =(14 s \alpha-180) \alpha<0
\end{aligned}
$$

and then it follows that

$$
h_{1} \geq \frac{g\left(s_{2}\right)}{720 s_{2}} \geq \frac{g(2 / \alpha)}{720 s_{2}}=\frac{1}{6 s_{2}}>0 .
$$

We obtain that for $s_{2} \in\left(0, \frac{2}{\alpha}\right]$,

$$
\begin{gathered}
h_{2}>\frac{\alpha^{2}}{6}-\frac{\alpha^{2}}{12}-\frac{5(s+1) \alpha^{2}}{720}=\left(\frac{1}{12}-\frac{s+1}{140}\right) \alpha^{2}>0, \text { and } \\
h_{3} \geq\left(\frac{1}{24}-\frac{s+2}{720}\right) \alpha^{3}>0(0<s \leq 6) .
\end{gathered}
$$

Hence, we have $h(x)>0$, and then setting $t=x^{1 / \alpha} u^{1 / \alpha}$, it follows that

$$
\begin{aligned}
\omega\left(s_{2}, x\right) & =x^{s-s_{2}} \sum_{n=1}^{\infty} g_{x}(n)<x^{s-s_{2}} \int_{0}^{\infty} g_{x}(t) d t \\
& =\alpha x^{s-s_{2}} \int_{0}^{\infty} \frac{t^{s_{2}-1}}{\left(x+t^{\alpha}\right)^{s}} d t=\int_{0}^{\infty} \frac{u^{s_{2}-1}}{(1+u)^{s}} d u \\
& =B\left(s_{2}, s-s_{2}\right)=k_{s}\left(s_{2}\right) .
\end{aligned}
$$

On the other hand, by (9), we have

$$
\begin{aligned}
\sum_{n=1}^{\infty} g_{x}(n) & =\int_{1}^{\infty} g_{x}(t) d t+\frac{1}{2} g_{x}(1)+\int_{1}^{\infty} P_{1}(t) g^{\prime}{ }_{x}(t) d t \\
& =\int_{1}^{\infty} g_{x}(t) d t+H(x),
\end{aligned}
$$

where

$$
H(x):=\frac{1}{2} g_{x}(1)+\int_{1}^{\infty} P_{1}(t) g_{x}^{\prime}(t) d t .
$$

We have obtained $\frac{1}{2} g_{x}(1)=\frac{\alpha}{2(x+1)^{s}}$ and

$$
g^{\prime}{ }_{x}(t)=-\frac{\alpha\left(\alpha s-\alpha s_{2}+1\right) t^{\alpha s_{2}-2}}{\left(x+t^{\alpha}\right)^{s}}+\frac{\alpha^{2} s x t^{\alpha s_{2}-2}}{\left(x+t^{\alpha}\right)^{s+1}} .
$$

For $s_{2} \in\left(0, \frac{2}{\alpha}\right] \cap(0, s), 0<s \leq 6$, by $(7)$, we have

$$
\begin{aligned}
& -\alpha\left(\alpha s-\alpha s_{2}+1\right) \int_{1}^{\infty} P_{1}(t) \frac{t^{\alpha s_{2}-2}}{\left(x+t^{\alpha}\right)^{s}} d t>0 \text { and } \\
& \alpha^{2} x s \int_{1}^{\infty} P_{1}(t) \frac{t^{\alpha s_{2}-2}}{\left(x+t^{\alpha}\right)^{s+1}} d t>-\frac{\alpha^{2} x s}{12(x+1)^{s+1}}>-\frac{\alpha^{2} s}{12(x+1)^{s}} .
\end{aligned}
$$

Hence, it follows that

$$
H(m)>\frac{\alpha}{2(x+1)^{s}}-\frac{\alpha^{2} s}{12(x+1)^{s}} \geq \frac{\alpha}{2(x+1)^{s}}-\frac{6 \alpha}{12(x+1)^{s}}=0,
$$

and then we obtain

$$
\begin{aligned}
\omega\left(\lambda_{2}, x\right) & =x^{s-s_{2}} \sum_{n=1}^{\infty} g_{x}(n)>x^{s-s_{2}} \int_{1}^{\infty} g_{x}(t) d t \\
& =x^{s-s_{2}} \int_{0}^{\infty} g_{x}(t) d t-x^{s-s_{2}} \int_{0}^{1} g_{x}(t) d t \\
& =k_{s}\left(s_{2}\right)\left[1-\frac{1}{k_{s}\left(s_{2}\right)} \int_{0}^{\frac{1}{x}} \frac{u^{s_{2}-1}}{(1+u)^{s}} d u\right]>0,
\end{aligned}
$$


where we set $O\left(\frac{1}{x^{s_{2}}}\right)=\frac{1}{k_{s}\left(s_{2}\right)} \int_{0}^{\frac{1}{x}} \frac{u^{s_{2}-1}}{(1+u)^{s}} d u$ satisfying

$$
0<\int_{0}^{\frac{1}{x}} \frac{u^{s_{2}-1}}{(1+u)^{s}} d u<\int_{0}^{\frac{1}{x}} u^{s_{2}-1} d u=\frac{1}{s_{2} x^{s_{2}}} .
$$

Therefore, inequalities (12) follow. Thus, Lemma 2 is proved.

Lemma 3. If $s \in(0,6], s_{1} \in(0, s), s_{2} \in\left(0, \frac{2}{\alpha}\right] \cap(0, s)$, then the following extended half-discrete Hardy-Hilbert's inequality holds:

$$
I=\int_{0}^{\infty} \sum_{n=1}^{\infty} \frac{a_{n} f(x)}{\left(x+n^{\alpha}\right)^{s}} d x \leq\left(\frac{1}{\alpha} k_{s}\left(s_{2}\right)\right)^{\frac{1}{p}}\left(k_{s}\left(s_{1}\right)\right)^{\frac{1}{q}} \cdot\left\{\int_{0}^{\infty} x^{p\left[1-\left(\frac{s-s_{2}}{p}+\frac{s_{1}}{q}\right)\right]-1} f^{p}(x) d x\right\}^{\frac{1}{p}}\left\{\sum_{n=1}^{\infty} n^{q\left[1-\alpha\left(\frac{s_{2}}{p}+\frac{s-s_{1}}{q}\right)\right]-1} a_{n}^{q}\right\}^{\frac{1}{q}}
$$

Proof. Setting $u=x / n^{\alpha}$, we obtain the following weight coefficient:

$$
\omega_{\alpha}\left(s_{1}, n\right):=n^{\alpha\left(s-s_{1}\right)} \int_{0}^{\infty} \frac{x^{s_{1}-1}}{\left(x+n^{\alpha}\right)^{s}} d x=\int_{0}^{\infty} \frac{u^{s_{1}-1}}{(u+1)^{s}} d u=k_{s}\left(s_{1}\right)(n \in \mathrm{N}) .
$$

By Hölder's inequality ([30]), we obtain

$$
\begin{gathered}
\left.I=\int_{0}^{\infty} \sum_{n=1}^{\infty} \frac{1}{\left(x+n^{\alpha}\right)^{s}} \frac{x^{\left(1-s_{1}\right) / q}\left(\alpha n^{\beta-1}\right)^{1 / p}}{n^{\alpha\left(1-s_{2}\right) / p}} f(x)\right]\left[\frac{n^{\alpha\left(1-s_{2}\right) / p}}{x^{\left(1-s_{1}\right) / q}\left(\alpha n^{\alpha-1}\right)^{1 / p}} a_{n}\right] d x \\
\leq\left[\sum_{n=1}^{\infty} \int_{0}^{\infty} \frac{\alpha}{\left(x+n^{\alpha}\right)^{s}} \frac{\left.x^{\left(1-s_{1}\right)(p-1)}\right)^{\alpha-1}}{n^{\alpha\left(1-s_{2}\right)}} f^{p}(x) d x\right]^{\frac{1}{p}} \\
\cdot\left[\int_{0}^{\infty} \sum_{n=1}^{\infty} \frac{1}{\left(x+n^{\alpha}\right)^{s}} \frac{n^{\alpha\left(1-s_{2}\right)(q-1)} a_{n}^{q}}{x^{1-s_{1}}\left(\alpha n^{\alpha-1}\right)^{q-1}} d x\right]^{\frac{1}{q}} \\
=\frac{1}{\alpha^{1 / p}}\left\{\int_{0}^{\infty} \omega_{\alpha}\left(s_{2}, x\right) x^{p\left[1-\left(\frac{s-s_{2}}{p}+\frac{s_{1}}{q}\right)\right]-1} f^{p}(x) d x\right\}^{\frac{1}{p}} \\
\cdot\left\{\sum_{n=1}^{\infty} \omega_{\alpha}\left(s_{1}, n\right) n^{q\left[1-\alpha\left(\frac{s-s_{1}}{q}+\frac{s_{2}}{p}\right)\right]-1} a_{n}^{q}\right\}^{\frac{1}{q}} .
\end{gathered}
$$

Thus, by (12) and (14), we get inequality (13). The proof of Lemma 3 is complete.

Remark 1. Fors $=\lambda+2 \in(2,6], \lambda \in(0,4], s_{1}=\lambda_{1}+1 \in(1, s), \lambda_{1} \in(0, \lambda+1)$,

$$
s_{2}=\lambda_{2}+1 \in\left(1, \frac{2}{\alpha}\right], \lambda_{2} \in\left(0, \frac{2}{\alpha}-1\right] \cap(0, \lambda+1),
$$

by replacing in (13), $f(x)$ and $a_{n}$ respectively with $F(x)$ and $A_{n}$, in view of (5), we have

$$
\begin{gathered}
\int_{0}^{\infty} \sum_{n=1}^{\infty} \frac{A_{n}}{\left(x+n^{\alpha}\right)^{\lambda+2}} F(x) d x<\left(\frac{1}{\alpha} k_{\lambda+2}\left(\lambda_{2}+1\right)\right)^{\frac{1}{p}}\left(k_{\lambda+2}\left(\lambda_{1}+1\right)\right)^{\frac{1}{q}} \\
\cdot\left[\int_{0}^{\infty} x^{-p\left(\frac{\lambda-\lambda_{2}}{p}+\frac{\lambda_{1}}{q}\right)-1} F^{p}(x) d x\right]^{\frac{1}{p}}\left\{\sum_{n=1}^{\infty} n^{q\left[1-\alpha\left(1+\frac{\lambda_{2}}{p}+\frac{\lambda-\lambda_{1}}{q}\right)\right]-1} A_{n}^{q}\right\}^{\frac{1}{q}} .
\end{gathered}
$$

Lemma 4. For $t>0$, we have

$$
\int_{0}^{\infty} e^{-t x} f(x) d x=t \int_{0}^{\infty} e^{-t x} F(x) d x
$$

and

$$
\sum_{n=1}^{\infty} e^{-t n^{\alpha}} a_{n} \leq t \sum_{n=1}^{\infty} e^{-t n^{\alpha}} A_{n} .
$$


Proof. Integrating by parts, in view of $F(x)=o\left(e^{t x}\right)(t>0 ; x \rightarrow \infty)$, it follows that

$$
\begin{aligned}
& \quad \int_{0}^{\infty} e^{-t x} f(x) d x=\int_{0}^{\infty} e^{-t x} d F(x)=\left.e^{-t x} F(x)\right|_{0} ^{\infty}-\int_{0}^{\infty} F(x) d e^{-t x} \\
& =\lim _{x \rightarrow \infty} e^{-t x} F(x)+t \int_{0}^{\infty} e^{-t x} F(x) d x=t \int_{0}^{\infty} e^{-t x} F(x) d x,
\end{aligned}
$$

and then we obtain inequality (16).

In view of $A_{n} e^{-t n^{\alpha}}=o(1)(n \rightarrow \infty)$, by Abel's summation by parts formula, we obtain

$$
\begin{aligned}
\sum_{n=1}^{\infty} e^{-t n^{\alpha}} a_{n} & =\lim _{n \rightarrow \infty} A_{n} e^{-t n^{\alpha}}+\sum_{n=1}^{\infty} A_{n}\left[e^{-t n^{\alpha}}-e^{-t(n+1)^{\alpha}}\right] \\
& =\sum_{n=1}^{\infty} A_{n}\left[e^{-t n^{\alpha}}-e^{-t(n+1)^{\alpha}}\right] .
\end{aligned}
$$

Since $1-e^{-t}<t(t>0)$ and for $\alpha \in(0,1]$,

$$
(n+1)^{\alpha}-n^{\alpha}-1=\alpha\left(n+\theta_{n}\right)^{\alpha-1}-1 \leq 0\left(\theta_{n} \in(0,1)\right),
$$

it follows that $e^{t\left[(n+1)^{\alpha}-n^{\alpha}-1\right]} \leq 1(t>0)$, which implies $e^{-t(n+1)^{\alpha}} \geq e^{-t\left(n^{\alpha}+1\right)}$. Thus, we obtain

$$
\begin{aligned}
\sum_{n=1}^{\infty} e^{-t n^{\alpha}} a_{n} & \leq \sum_{n=1}^{\infty} A_{n}\left[e^{-t n^{\alpha}}-e^{-t\left(n^{\alpha}+1\right)}\right] \\
& =\left(1-e^{-t}\right) \sum_{n=1}^{\infty} A_{n} e^{-t n^{\alpha}} \leq t \sum_{n=1}^{\infty} A_{n} e^{-t n^{\alpha}}
\end{aligned}
$$

Hence, the inequality (17) is derived. This completes the proof of Lemma 4.

\section{Main Results}

Theorem 1. The following half-discrete Hilbert-type inequality holds true:

$$
\begin{aligned}
I:= & \int_{0}^{\infty} \sum_{n=1}^{\infty} \frac{a_{n}}{\left(x+n^{\alpha}\right)^{\lambda}} f(x) d x<\frac{\Gamma(\lambda+2)}{\Gamma(\lambda)}\left(\frac{1}{\alpha} k_{\lambda+2}\left(\lambda_{2}+1\right)\right)^{\frac{1}{p}}\left(k_{\lambda+2}\left(\lambda_{1}+1\right)\right)^{\frac{1}{q}} \\
& \cdot\left[\int_{0}^{\infty} x^{-p\left(\frac{\lambda-\lambda_{2}}{p}+\frac{\lambda_{1}}{q}\right)-1} F^{p}(x) d x\right]^{\frac{1}{p}}\left\{\sum_{n=1}^{\infty} n^{q\left[1-\alpha\left(1+\frac{\lambda-\lambda_{1}}{q}+\frac{\lambda_{2}}{p}\right)\right]-1} A_{n}^{q}\right\}^{\frac{1}{q}},
\end{aligned}
$$

where $\Gamma(\cdot)$ is the gamma function.

In particular, for $\lambda_{1}+\lambda_{2}=\lambda$, which implies that $k_{\lambda}\left(\lambda_{1}\right)=B\left(\lambda_{1}, \lambda_{2}\right)$ and

$$
\begin{aligned}
& \frac{\lambda-\lambda_{2}}{p}+\frac{\lambda_{1}}{q}=\frac{\lambda_{1}}{p}+\frac{\lambda_{1}}{q}=\lambda_{1}, \frac{\lambda-\lambda_{1}}{q}+\frac{\lambda_{2}}{p}=\frac{\lambda_{2}}{q}+\frac{\lambda_{2}}{p}=\lambda_{2}, \\
& 0<\int_{0}^{\infty} x^{-p \lambda_{1}-1} F^{p}(x) d x<\infty, 0<\sum_{n=1}^{\infty} n^{q\left[1-\alpha\left(\lambda_{2}+1\right)\right]-1} A_{n}^{q}<\infty,
\end{aligned}
$$

and hence, the following inequality holds:

$$
\begin{aligned}
I & =\int_{0}^{\infty} \sum_{n=1}^{\infty} \frac{a_{n}}{\left(x+n^{\alpha}\right)^{\lambda}} f(x) d x<\frac{\lambda_{1} \lambda_{2}}{\alpha^{1 / p}} k_{\lambda}\left(\lambda_{1}\right) \\
& \cdot\left(\int_{0}^{\infty} x^{-p \lambda_{1}-1} F^{p}(x) d x\right)^{\frac{1}{p}}\left\{\sum_{n=1}^{\infty} n^{q\left[1-\alpha\left(1+\lambda_{2}\right)\right]-1} A_{n}^{q}\right\}^{\frac{1}{q}} .
\end{aligned}
$$

Proof. Since

$$
\frac{1}{\left(x+n^{\alpha}\right)^{\lambda}}=\frac{1}{\Gamma(\lambda)} \int_{0}^{\infty} t^{\lambda-1} e^{-\left(x+n^{\alpha}\right) t} d t
$$


it follows that

$$
\begin{aligned}
I= & \frac{1}{\Gamma(\lambda)} \int_{0}^{\infty} \sum_{n=1}^{\infty} a_{n} f(x) \int_{0}^{\infty} t^{\lambda-1} e^{-\left(x+n^{\alpha}\right) t} d t d x \\
& =\frac{1}{\Gamma(\lambda)} \int_{0}^{\infty} t^{\lambda-1} \int_{0}^{\infty} e^{-x t} f(x) d x \sum_{n=1}^{\infty} e^{-n^{\alpha} t} a_{n} d t \\
& \leq \frac{1}{\Gamma(\lambda)} \int_{0}^{\infty} t^{\lambda+1} \int_{0}^{\infty} e^{-x t} F(x) d x \sum_{n=1}^{\infty} e^{-n^{\alpha} t} A_{n} d t \\
& =\frac{1}{\Gamma(\lambda)} \int_{0}^{\infty} \sum_{n=1}^{\infty} A_{n} F(x) \int_{0}^{\infty} t^{\lambda+1} e^{-\left(x+n^{\alpha}\right) t} d t d x \\
& =\frac{\Gamma(\lambda+2)}{\Gamma(\lambda)} \int_{0}^{\infty} \sum_{n=1}^{\infty} \frac{A_{n}}{\left(x+n^{\alpha}\right)^{\lambda+2}} F(x) d x .
\end{aligned}
$$

By inequality (15), we obtain inequality (18). Thus, Theorem 1 is proved.

Remark 2. Putting $\alpha=1, \lambda_{1} \in(0, \lambda+1), \lambda_{2} \in(0,1] \cap(0, \lambda+1)$ in (18), we have the following half-discrete Hilbert-type inequality:

$$
\begin{gathered}
\int_{0}^{\infty} \sum_{n=1}^{\infty} \frac{a_{n}}{(x+n)^{\lambda}} f(x) d x<\frac{\Gamma(\lambda+2)}{\Gamma(\lambda)}\left(k_{\lambda+2}\left(\lambda_{2}+1\right)\right)^{\frac{1}{p}}\left(k_{\lambda+2}\left(\lambda_{1}+1\right)\right)^{\frac{1}{q}} \\
\cdot\left[\int_{0}^{\infty} x^{-p\left(\frac{\lambda-\lambda_{2}}{p}+\frac{\lambda_{1}}{q}\right)-1} F^{p}(x) d x\right]^{\frac{1}{p}}\left[\sum_{n=1}^{\infty} n^{-q\left(\frac{\lambda-\lambda_{1}}{q}+\frac{\lambda_{2}}{p}\right)-1} A_{n}^{q}\right]^{\frac{1}{q}}
\end{gathered}
$$

Theorem 2. For $\lambda_{1} \in(0, \lambda), \lambda_{2} \in(0,1] \cap(0, \lambda)$, the constant factor

$$
\frac{\Gamma(\lambda+2)}{\Gamma(\lambda)}\left(k_{\lambda+2}\left(\lambda_{2}+1\right)\right)^{\frac{1}{p}}\left(k_{\lambda+2}\left(\lambda_{1}+1\right)\right)^{\frac{1}{q}}
$$

in (20) is the best possible if and only if $\lambda_{1}+\lambda_{2}=\lambda(\in(0,4])$.

Proof. Firstly, we shall prove that under the condition $\lambda_{1}+\lambda_{2}=\lambda(\in(0,4])$, the constant factor given in (20) is the best possible.

If $\lambda_{1}+\lambda_{2}=\lambda(\in(0,4])\left(\lambda_{1} \in(0, \lambda), \lambda_{2} \in(0,1] \cap(0, \lambda)\right)$, then we obtain

$$
\begin{aligned}
& k_{\lambda+2}\left(\lambda_{2}+1\right)=\int_{0}^{\infty} \frac{u^{\left(\lambda_{2}+1\right)-1}}{(1+u)^{\lambda+2}} d u=B\left(\lambda_{2}+1,(\lambda+2)-\left(\lambda_{2}+1\right)\right) \\
& =B\left(\lambda_{2}+1, \lambda_{1}+1\right)=B\left(\lambda_{1}+1,(\lambda+2)-\left(\lambda_{1}+1\right)\right) \\
& =k_{\lambda+2}\left(\lambda_{1}+1\right)=\frac{\Gamma\left(\lambda_{1}+1\right) \Gamma\left(\lambda_{2}+1\right)}{\Gamma(\lambda+2)}=\frac{\lambda_{1} \lambda_{2} \Gamma\left(\lambda_{1}\right) \Gamma\left(\lambda_{2}\right)}{\Gamma(\lambda+2)} \text {, } \\
& \frac{\Gamma(\lambda+2)}{\Gamma(\lambda)}\left(k_{\lambda+2}\left(\lambda_{2}+1\right)\right)^{\frac{1}{p}}\left(k_{\lambda+2}\left(\lambda_{1}+1\right)\right)^{\frac{1}{q}} \\
& =\frac{\Gamma(\lambda+2)}{\Gamma(\lambda)} k_{\lambda+2}\left(\lambda_{1}+1\right)=\frac{\Gamma(\lambda+2)}{\Gamma(\lambda)} \lambda_{1} \lambda_{2} \frac{\Gamma\left(\lambda_{1}\right) \Gamma\left(\lambda_{2}\right)}{\Gamma(\lambda+2)} \\
& =\lambda_{1} \lambda_{2} \frac{\Gamma\left(\lambda_{1}\right) \Gamma\left(\lambda_{2}\right)}{\Gamma(\lambda)}=\lambda_{1} \lambda_{2} B\left(\lambda_{1}, \lambda_{2}\right) \text {, }
\end{aligned}
$$

and $\frac{\lambda-\lambda_{2}}{p}+\frac{\lambda_{1}}{q}=\lambda_{1}, \frac{\lambda-\lambda_{1}}{q}+\frac{\lambda_{2}}{p}=\lambda_{2}$. Thus, we can reduce (20) to the following:

$$
\begin{aligned}
& \int_{0}^{\infty} \sum_{n=1}^{\infty} \frac{a_{n}}{(x+n)^{\lambda}} f(x) d x \\
& <\lambda_{1} \lambda_{2} B\left(\lambda_{1}, \lambda_{2}\right)\left(\int_{0}^{\infty} x^{-p \lambda_{1}-1} F^{p}(x) d x\right)^{\frac{1}{p}}\left(\sum_{n=1}^{\infty} n^{-q \lambda_{2}-1} A_{n}^{q}\right)^{\frac{1}{q}} .
\end{aligned}
$$

For any $0<\varepsilon<\min \left\{p \lambda_{1}, q \lambda_{2}\right\}$, we set the following function and sequence:

$$
\widetilde{f}(x):=\left\{\begin{array}{l}
0,0<x<1, \\
x^{\lambda_{1}-\frac{\varepsilon}{p}-1}, x \geq 1
\end{array}, \widetilde{a}_{n}:=n^{\lambda_{2}-\frac{\varepsilon}{q}-1}(n \in \mathrm{N}) .\right.
$$


Then it follows that

$$
\begin{aligned}
& \widetilde{F}(x):=\int_{0}^{x} \widetilde{f}(t) d t \leq\left\{\begin{array}{l}
0,0<x<1 \\
\frac{1}{\lambda_{1}-\frac{\varepsilon}{p}} x^{\lambda_{1}-\frac{\varepsilon}{p}}, x \geq 1
\end{array}\right. \\
& \widetilde{A_{n}}:=\sum_{k=1}^{n} \widetilde{a}_{k}=\sum_{k=1}^{n} k^{\lambda_{2}-\frac{\varepsilon}{q}-1}<\int_{0}^{n} t^{\lambda_{2}-\frac{\varepsilon}{q}-1} d t=\frac{1}{\lambda_{2}-\frac{\varepsilon}{q}} n^{\lambda_{2}-\frac{\varepsilon}{q}}(n \in \mathrm{N}) .
\end{aligned}
$$

If there exists a positive constant

$$
M \leq \lambda_{1} \lambda_{2} B\left(\lambda_{1}, \lambda_{2}\right)
$$

such that (21) is valid when replacing $\lambda_{1} \lambda_{2} B\left(\lambda_{1}, \lambda_{2}\right)$ by $M$, then in particular, by substitution of $f(x)=\widetilde{f}(x), a_{n}=\widetilde{a}_{n}, F(x)=\widetilde{F}(x)$ and $A_{n}=\widetilde{A}_{n}$ in (21), we have

$$
\widetilde{I}:=\int_{0}^{\infty} \sum_{n=1}^{\infty} \frac{\widetilde{a}_{n} \widetilde{f}(x)}{(x+n)^{\lambda}} d x<M\left(\int_{0}^{\infty} x^{-p \lambda_{1}-1} \widetilde{F}^{p}(x) d x\right)^{\frac{1}{p}}\left(\sum_{n=1}^{\infty} n^{-q \lambda_{2}-1} \widetilde{A}_{n}^{q}\right)^{\frac{1}{q}} .
$$

By (22) and the decreasingness property of series, we obtain

$$
\begin{aligned}
\widetilde{I}< & M \frac{1}{\lambda_{1}-\frac{\varepsilon}{p}}\left(\int_{1}^{\infty} x^{-p \lambda_{1}-1} x^{p \lambda_{1}-\varepsilon} d x\right)^{\frac{1}{p}} \frac{1}{\lambda_{2}-\frac{\varepsilon}{q}}\left(\sum_{n=1}^{\infty} n^{-q \lambda_{2}-1} n^{q \lambda_{2}-\varepsilon}\right)^{\frac{1}{q}} \\
& =M\left(\frac{1}{\lambda_{1}-\frac{\varepsilon}{p}}\right)\left(\frac{1}{\lambda_{2}-\frac{\varepsilon}{q}}\right)\left(\int_{1}^{\infty} x^{-\varepsilon-1} d x\right)^{\frac{1}{p}}\left(1+\sum_{n=2}^{\infty} n^{-\varepsilon-1}\right)^{\frac{1}{q}} \\
& <M\left(\frac{1}{\lambda_{1}-\frac{\varepsilon}{p}}\right)\left(\frac{1}{\lambda_{2}-\frac{\varepsilon}{q}}\right)\left(\int_{1}^{\infty} x^{-\varepsilon-1} d x\right)^{\frac{1}{p}}\left(1+\int_{1}^{\infty} y^{-\varepsilon-1} d y\right)^{\frac{1}{q}} \\
& =\frac{M}{\varepsilon}\left(\frac{1}{\lambda_{1}-\frac{\varepsilon}{p}}\right)\left(\frac{1}{\lambda_{2}-\frac{\varepsilon}{q}}\right)(\varepsilon+1)^{\frac{1}{q}}
\end{aligned}
$$

By (12) (for $\alpha=1)$, setting $\widetilde{\lambda}_{2}:=\lambda_{2}-\frac{\varepsilon}{q} \in(0,1) \cap(0, \lambda)\left(0<\widetilde{\lambda}_{1}:=\lambda_{2}+\frac{\varepsilon}{q}=\lambda-\widetilde{\lambda}_{2}<\lambda\right)$, we find

$$
\begin{aligned}
\widetilde{I} & =\int_{1}^{\infty}\left[x^{\lambda_{1}-\frac{\varepsilon}{q}} \sum_{n=1}^{\infty} \frac{1}{(x+n)^{\lambda}} n^{\left(\lambda_{2}-\frac{\varepsilon}{q}\right)-1}\right] x^{-\varepsilon-1} d x \\
& =\int_{1}^{\infty} \omega_{1}\left(\widetilde{\lambda}_{2}, x\right) x^{-\varepsilon-1} d x>B\left(\widetilde{\lambda}_{1}, \widetilde{\lambda}_{2}\right) \int_{1}^{\infty}\left(1-O\left(x^{-\widetilde{\lambda}_{2}}\right)\right) x^{-\varepsilon-1} d x \\
& =B\left(\widetilde{\lambda}_{1}, \widetilde{\lambda}_{2}\right)\left(\int_{1}^{\infty} x^{-\varepsilon-1} d x-\int_{1}^{\infty} O\left(x^{-\lambda_{2}-\frac{\varepsilon}{p}-1}\right) d x\right) \\
& =\frac{1}{\varepsilon} B\left(\lambda_{1}+\frac{\varepsilon}{q}, \lambda_{2}-\frac{\varepsilon}{q}\right)(1-\varepsilon O(1)) .
\end{aligned}
$$

Then, in virtue of the above results, we have

$$
B\left(\lambda_{1}+\frac{\varepsilon}{q}, \lambda_{2}-\frac{\varepsilon}{q}\right)(1-\varepsilon O(1))<\varepsilon \widetilde{I}<M\left(\frac{1}{\lambda_{1}-\frac{\varepsilon}{p}}\right)\left(\frac{1}{\lambda_{2}-\frac{\varepsilon}{q}}\right)(\varepsilon+1)^{\frac{1}{q}} .
$$

For $\varepsilon \rightarrow 0^{+}$, in view of the continuity of the beta function, we obtain

$$
\lambda_{1} \lambda_{2} B\left(\lambda_{1}, \lambda_{2}\right) \leq M
$$

Hence, $M=\lambda_{1} \lambda_{2} B\left(\lambda_{1}, \lambda_{2}\right)$ is the best possible constant factor of (21).

Secondly, we need to prove that if the constant factor given in (20) is the best possible, then $\lambda_{1}+\lambda_{2}=\lambda(\in(0,4])$. 
Setting $\hat{\lambda}_{1}:=\frac{\lambda-\lambda_{2}}{p}+\frac{\lambda_{1}}{q}, \hat{\lambda}_{2}:=\frac{\lambda-\lambda_{1}}{q}+\frac{\lambda_{2}}{p}$, we reduce (20) to the following:

$$
\begin{gathered}
\int_{0}^{\infty} \sum_{n=1}^{\infty} \frac{a_{n}}{(x+n)^{\lambda}} f(x) d x<\frac{\Gamma(\lambda+2)}{\Gamma(\lambda)}\left(k_{\lambda+2}\left(\lambda_{2}+1\right)\right)^{\frac{1}{p}}\left(k_{\lambda+2}\left(\lambda_{1}+1\right)\right)^{\frac{1}{q}} \\
\cdot\left(\int_{0}^{\infty} x^{-p \hat{\lambda}_{1}-1} F^{p}(x) d x\right)^{\frac{1}{p}}\left(\sum_{n=1}^{\infty} n^{-q \hat{\lambda}_{2}-1} A_{n}^{q}\right)^{\frac{1}{q}}
\end{gathered}
$$

We obtain

$$
\begin{aligned}
& \hat{\lambda}_{1}+\hat{\lambda}_{2}=\frac{\lambda-\lambda_{2}}{p}+\frac{\lambda_{1}}{q}+\frac{\lambda-\lambda_{1}}{q}+\frac{\lambda_{2}}{p}=\lambda, \\
& 0<\hat{\lambda}_{1}, \hat{\lambda}_{2}<\frac{\lambda}{p}+\frac{\lambda}{q}=\lambda,
\end{aligned}
$$

and then we have

$$
\hat{\lambda}_{1} \hat{\lambda}_{2} k_{\lambda}\left(\hat{\lambda}_{1}\right)=\hat{\lambda}_{1} \hat{\lambda}_{2} B\left(\hat{\lambda}_{1}, \hat{\lambda}_{2}\right) \in \mathrm{R}_{+}=(0, \infty)
$$

If the constant factor

$$
\frac{\Gamma(\lambda+2)}{\Gamma(\lambda)}\left(k_{\lambda+2}\left(\lambda_{2}+1\right)\right)^{\frac{1}{p}}\left(k_{\lambda+2}\left(\lambda_{1}+1\right)\right)^{\frac{1}{q}}
$$

in (20) (or (23)) is the best possible, then by (21), the unified best possible constant factor must be $\hat{\lambda}_{1} \hat{\lambda}_{2} B\left(\hat{\lambda}_{1}, \hat{\lambda}_{2}\right) \in \mathrm{R}_{+}$, namely,

$$
\begin{aligned}
& \frac{\Gamma(\lambda+2)}{\Gamma(\lambda)}\left(k_{\lambda+2}\left(\lambda_{2}+1\right)\right)^{\frac{1}{p}}\left(k_{\lambda+2}\left(\lambda_{1}+1\right)\right)^{\frac{1}{q}} \\
& =\hat{\lambda}_{1} \hat{\lambda}_{2} B\left(\hat{\lambda}_{1}, \hat{\lambda}_{2}\right)=\frac{\Gamma(\lambda+2)}{\Gamma(\lambda)} k_{\lambda+2}\left(\hat{\lambda}_{1}+1\right) .
\end{aligned}
$$

It follows that

$$
k_{\lambda+2}\left(\hat{\lambda}_{1}+1\right)=\left(k_{\lambda+2}\left(\lambda_{2}+1\right)\right)^{\frac{1}{p}}\left(k_{\lambda+2}\left(\lambda_{1}+1\right)\right)^{\frac{1}{q}}
$$

By Hölder's inequality with weight, we obtain

$$
\begin{aligned}
& k_{\lambda+2}\left(\hat{\lambda}_{1}+1\right)=k_{\lambda+2}\left(\frac{\lambda-\lambda_{2}}{p}+\frac{\lambda_{1}}{q}+1\right) \\
= & \int_{0}^{\infty} \frac{1}{(1+u)^{\lambda+2}} u^{\frac{\lambda-\lambda_{2}}{p}+\frac{\lambda_{1}}{q}} d u=\int_{0}^{\infty} \frac{1}{(1+u)^{\lambda+2}}\left(u^{\frac{\lambda-\lambda_{2}}{p}}\right)\left(u^{\frac{\lambda_{1}}{q}}\right) d u \\
\leq & {\left[\int_{0}^{\infty} \frac{1}{(1+u)^{\lambda+2}} u^{\lambda-\lambda_{2}} d u\right]^{\frac{1}{p}}\left[\int_{0}^{\infty} \frac{1}{(1+u)^{\lambda+2}} u^{\lambda_{1}} d u\right]^{\frac{1}{q}} } \\
= & {\left[\int_{0}^{\infty} \frac{1}{(1+v)^{\lambda+2}} v^{\left(\lambda_{2}+1\right)-1} d v\right]^{\frac{1}{p}}\left[\int_{0}^{\infty} \frac{1}{(1+u)^{\lambda+2}} u^{\left(\lambda_{1}+1\right)-1} d u\right]^{\frac{1}{q}} } \\
= & \left(k_{\lambda+2}\left(\lambda_{2}+1\right)\right)^{\frac{1}{p}}\left(k_{\lambda+2}\left(\lambda_{1}+1\right)\right)^{\frac{1}{q}} .
\end{aligned}
$$

We observe that (24) keeps the form of equality if and only if there exist constants $A$ and $B$ such that they are not all zero and ([30])

$$
A u^{\lambda-\lambda_{2}}=B u^{\lambda_{1}} \text { a.e. in } \mathrm{R}_{+} .
$$

Assuming that $A \neq 0$, we have

$$
u^{\lambda-\lambda_{2}-\lambda_{1}}=\frac{B}{A} \text { a.e. in } \mathrm{R}_{+},
$$

and then one has $\lambda-\lambda_{2}-\lambda_{1}=0$, namely, $\lambda_{1}+\lambda_{2}=\lambda(\in(0,4])$. This completes the proof of Theorem 2. $\square$ 
Remark 3. (i) For $\lambda=1, \lambda_{1}=\frac{1}{r}, \lambda_{2}=\frac{1}{s}\left(r>1, \frac{1}{r}+\frac{1}{s}=1\right)$ in (21), we have the following half-discrete Hilbert-type inequality with the best possible constant factor $\frac{\pi}{r s \sin (\pi / r)}$ :

$$
\int_{0}^{\infty} \sum_{n=1}^{\infty} \frac{a_{n} f(x)}{x+n} d x<\frac{\pi}{r s \sin (\pi / r)}\left(\int_{0}^{\infty} x^{-\frac{p}{r}-1} F^{p}(x) d x\right)^{\frac{1}{p}}\left(\sum_{n=1}^{\infty} n^{-\frac{q}{s}-1} A_{n}^{q}\right)^{\frac{1}{q}} .
$$

In particular, for $r=p, s=q$, we get

$$
\int_{0}^{\infty} \sum_{n=1}^{\infty} \frac{a_{n} f(x)}{x+n} d x<\frac{\pi}{p q \sin (\pi / p)}\left(\int_{0}^{\infty} x^{-2} F^{p}(x) d x\right)^{\frac{1}{p}}\left(\sum_{n=1}^{\infty} n^{-2} A_{n}^{q}\right)^{\frac{1}{q}} ;
$$

for $r=q, s=p$, we obtain

$$
\int_{0}^{\infty} \sum_{n=1}^{\infty} \frac{a_{n} f(x)}{x+n} d x<\frac{\pi}{p q \sin (\pi / p)}\left(\int_{0}^{\infty} x^{-p} F^{p}(x) d x\right)^{\frac{1}{p}}\left(\sum_{n=1}^{\infty} n^{-q} A_{n}^{q}\right)^{\frac{1}{q}} .
$$

(ii) For $\lambda=2, \lambda_{1}=\lambda_{2}=1$ in (21), we have the following half-discrete Hilbert-type inequality with the best possible constant factor 1 :

$$
\int_{0}^{\infty} \sum_{n=1}^{\infty} \frac{a_{n}}{(x+n)^{2}} f(x) d x<\left(\int_{0}^{\infty} \frac{F^{p}(x)}{x^{p+1}} d x\right)^{\frac{1}{p}}\left(\sum_{n=1}^{\infty} \frac{A_{n}^{q}}{n^{q+1}}\right)^{\frac{1}{q}}
$$

\section{Conclusions}

In this paper, by means of the weight coefficients, the idea of introduced parameters, the Euler-Maclaurin summation formula and Abel's summation by parts formula, a new half-discrete Hilbert-type inequality involving the variable upper limit integral and partial sums is given in Theorem 1. As an application, an inequality obtained from the special case of the half-discrete Hilbert-type inequality is investigated in Theorem 2, we obtained the equivalent conditions of the best possible constant factor related to several parameters. The lemmas and theorems proved in this paper reveal some new and interesting properties of this type of inequalities.

Author Contributions: J.L. and S.W. participated in the design of the study and performed the numerical analysis. B.Y. carried out the mathematical studies, participated in the research team and drafted the manuscript. All authors contributed equally and significantly in this paper. All authors have read and agreed to the published version of the manuscript.

Funding: This work is supported by the National Natural Science Foundation (Nos. 61772140, 11401113), the Science and Technology Planning Project Item of Guangzhou City (No. 201707010229) and the Characteristic Innovation Project (Natural Science) of Guangdong Province (No. 2017KTSCX133).

Acknowledgments: The authors are grateful to the reviewers for their valuable comments and suggestions to improve the quality of the manuscript.

Conflicts of Interest: The authors declare no conflict of interest.

\section{References}

1. Hardy, G.H.; Littlewood, J.E.; Polya, G. Inequalities; Cambridge University Press: Cambridge, UK, 1934.

2. Krnić, M.; Pečarić, J. Extension of Hilbert's inequality. J. Math. Anal. Appl. 2006, 324, 150-160. [CrossRef]

3. Adiyasuren, V.; Batbold, T.; Azar, L.E. A new discrete Hilbert-type inequality involving partial sums. J. Inequalities Appl. 2019, 2019, 127. [CrossRef]

4. Yang, B.C. On a generalization of Hilbert double series theorem. J. Nanjing Univ. Math. 2001, 18, 145-152.

5. Yang, B.C. The Norm of Operator and Hilbert-Type Inequalities; Science Press: Beijing, China, 2009. 
6. Krnić, M.; Pečarić, J. General Hilbert's and Hardy's inequalities. Math. Inequalities Appl. 2005, 8, $29-51$. [CrossRef]

7. Perić, I.; Vuković, P. Multiple Hilbert's type inequalities with a homogeneous kernel. Banach J. Math. Anal. 2011, 5, 33-43. [CrossRef]

8. Huang, Q.L. A new extension of Hardy-Hilbert-type inequality. J. Inequalities Appl. 2015, 2015, 397. [CrossRef]

9. He, B. A multiple Hilbert-type discrete inequality with a new kernel and best possible constant factor. $J$. Math. Anal. Appl. 2015, 431, 889-902. [CrossRef]

10. Xu, J.S. Hardy-Hilbert's inequalities with two parameters. Adv. Math. 2007, 36, 63-76.

11. Xie, Z.T.; Zeng, Z.; Sun, Y.F. A new Hilbert-type inequality with the homogeneous kernel of degree -2 . Adv. Appl. Math. Sci. 2013, 12, 391-401.

12. Zhen, Z.; Raja Rama Gandhi, K.; Xie, Z.T. A new Hilbert-type inequality with the homogeneous kernel of degree -2 and with the integral. Bull. Math. Sci. Appl. 2014, 7, 9-17.

13. Xin, D.M. A Hilbert-type integral inequality with the homogeneous kernel of zero degree. Math. Theory Appl. 2010, 30, 70-74.

14. Azar, L.E. The connection between Hilbert and Hardy inequalities. J. Inequalities Appl. 2013, $2013,452$. [CrossRef]

15. Adiyasuren, V.; Batbold, T.; Krnić, M. Hilbert-type inequalities involving differential operators, the best constants and applications. Math. Inequalities Appl. 2015, 18, 111-124. [CrossRef]

16. Rassias, M.T.; Yang, B.C. On half-discrete Hilbert's inequality. Appl. Math. Comput. 2013, 220, 75-93. [CrossRef]

17. Yang, B.C.; Krnić, M. A half-discrete Hilbert-type inequality with a general homogeneous kernel of degree 0 . J. Math. Inequalities 2012, 6, 401-417.

18. Rassias, M.T.; Yang, B.C. A multidimensional half-Discrete Hilbert-Type inequality and the Riemann zeta function. Appl. Math. Comput. 2013, 225, 263-277. [CrossRef]

19. Rassias, M.T.; Yang, B.C. On a multidimensional half-discrete Hilbert-Type inequality related to the hyperbolic cotangent function. Appl. Math. Comput. 2013, 242, 800-813. [CrossRef]

20. Rassias, M.T.; Yang, B.C. On an equivalent property of a reverse Hilbert-type integral inequality related to the extended Hurwitz-zeta function. J. Math. Inequalities 2019, 13, 315-334. [CrossRef]

21. Hong, Y.; Wen, Y.M. A necessary and sufficient condition of that Hilbert type series inequality with homogeneous kernel has the best constant factor. Ann. Math. 2016, 37, 329-336.

22. Hong, Y. On the structure character of Hilbert's type integral inequality with homogeneous kernel and application. J. Jilin Univ. Sci. Ed. 2017, 55, 189-194.

23. Hong, Y.; Huang, Q.L.; Yang, B.C.; Liao, J.Q. The necessary and sufficient conditions for the existence of a kind of Hilbert-type multiple integral inequality with the non -homogeneous kernel and its applications. J. Inequalities Appl. 2017, 2017, 316. [CrossRef]

24. Xin, D.M.; Yang, B.C.; Wang, A.Z. Equivalent property of a Hilbert-type integral inequality related to the beta function in the whole plane. J. Funct. Spaces 2018, 2018, 1-8. [CrossRef]

25. Hong, Y.; He, B.; Yang, B.C. Necessary and sufficient conditions for the validity of Hilbert type integral inequalities with a class of quasi-homogeneous kernels and its application in operator theory. J. Math. Inequalities 2018, 12, 777-788. [CrossRef]

26. Huang, Z.X.; Yang, B.C. Equivalent property of a half-discrete Hilbert's inequality with parameters. J. Inequalities Appl. 2018, 2018, 333. [CrossRef] [PubMed]

27. Wang, A.Z.; Yang, B.C.; Chen, Q. Equivalent properties of a reverse's half-discret Hilbert's inequality. J. Inequalities Appl. 2019, 2019, 279. [CrossRef]

28. Yang, B.C.; Wu, S.H.; Wang, A.Z. On a reverse half-discrete Hardy-Hilbert's inequality with parameters. Mathematics 2019, 7, 1054. [CrossRef]

29. Yang, B.C.; Wu, S.H.; Liao, J.Q. On a new extended Hardy-Hilbert's inequality with parameters. Mathematics 2020, 8, 73. [CrossRef]

30. Kuang, J.C. Applied Inequalities; Shangdong Science and Technology Press: Jinan, China, 2004.

(C) 2020 by the authors. Licensee MDPI, Basel, Switzerland. This article is an open access article distributed under the terms and conditions of the Creative Commons Attribution (CC BY) license (http://creativecommons.org/licenses/by/4.0/). 